\title{
Wavelet and Wavelet Packet Data Compression of Power System Disturbances
}

\author{
Non-member Effrina Yanti Hamid \\ Member \\ Member \\ Member
}

\author{
(Osaka University) \\ (Osaka University) \\ (Kansai Electric Power Co.) \\ (Kansai Electric Instrument Co.)
}

This paper introduces a compression technique for power disturbance data via discrete wavelet transform (DWT) and wavelet packet transform (WPT). The data compression leads to a potential application for remote power protection and power quality monitoring. The compression technique is performed through signal decomposition up to a certain level, thresholding of wavelet coefficients, and signal reconstruction. The choice of which wavelet to use for the compression is of critical importance, because the wavelet affects reconstructed signal quality and the design of the system as a whole. The Minimum Description Length (MDL) criterion is proposed for the selection of an appropriate wavelet filter. This criterion permits to select not only the suitable wavelet filter but also the best number of wavelet retained coefficients for signal reconstruction. The experimental study has been carried out for a single-phase to ground fault event, and the data compression results of using the suitable wavelet filter show that the compression ratios are varied from $2 \%$ to $11 \%$ and are reduced to more than a half of those values by implementing an additional lossless coding.

Keywords: Data Compression, Power System Disturbances, Wavelets, Wavelet Packets

\section{Introduction}

The transients due to ground faults, load switchings, and other disturbances may cover a broad frequency spectrum. A single captured event recorded for several seconds using monitoring instruments having tens to hundreds $\mathrm{KHz}$ sampling rate can produce kilo- to megabytes of data. As a result for several captured events, the volume of the generated and maintained data increase significantly, which lead to a high cost in storing and transmitting such data. Therefore, it is necessary to develop an effective compression technique which has capability to reduce the volume of data necessary for storing and to speed up the transmitted data for remote monitoring (Mehta and Russel, 1989; Santoso et al, 1997; Littler and Morrow, 1999).

Wavelet and wavelet packet transforms have recently emerged as powerful tools for a broad range of applications, signal compression in particular (Santoso et al, 1997; Littler and Morrow, 1999; Hilton, 1997; Walczak and Massart, 1997). The wavelet transform has good localization in both frequency and time domains, having fine frequency resolution and coarse time resolutions at lower frequency, and coarse frequency resolution and fine time resolution at higher frequency. It makes the wavelet transform suitable for time-frequency analysis. In data compression, the wavelet transform is used to exploit the redundancy in the signal. The performance of a wavelet transform for data compression lies in its ability in concentrating a large percentage of total signal energy in a few coefficients (Coifmann and
Wickerhauser, 1992). After the original signal is transformed into the wavelet coefficients, many coefficients are so small so that these coefficients can be omitted without losing significant information after the signal is reconstructed.

During the last three years, power disturbance data compression using wavelet and wavelet packet transforms have been proposed (Santoso et al, 1997; Littler and Morrow, 1999). The choice of which wavelet to use in compression system plays an important role, because the wavelet affects reconstructed signal quality and the design of the system as a whole. Compared with the actual compression performance of several different wavelets, the previous authors (Santoso et al, 1997; Littler and Morrow, 1999) choose only a specific wavelet filter. Improper choice of filter can produce distortions in the reconstructed signal. The previous authors also used a fix thresholding value to suppress the noise for the compression. However, in the real condition the noise level is difficult to estimate. Improper choice of filter and threshold setting can cause not optimum compression ratio. An algorithm to optimize the efficiency of compression in the wavelet domain called the Minimum Description Length (MDL) has been proposed (Saito, 1994). The algorithm permits one to select the suitable wavelet filter and the best number of wavelet retained coefficients of a signal, and it is free from threshold selection.

In this paper, we propose a data compression method based on wavelet and wavelet packet for power system disturbances. The method includes the selection 
of wavelet filter using the MDL criterion to optimize the compression technique. We evaluate several wavelet filters and compare their performances. Although there are many types of wavelet filters, we restrict ourselves to the Daubechies, Coiflets and Symlets families with a certain level of decomposition. In addition, the results from this wavelet-based compression method are then combined with a lossless coding e.g. Huffman, Lempel-Ziv-Welch (LZW), or LempelZiv-Haruyasu (LZH) to get more effective compression (Littler and Morrow, 1999).

\section{Wavelet}

2.1 Discrete Wavelet Transform The wavelet transform of a discrete input data sequence $f=\left\{f_{n}\right\}=$ $\left\{f_{0}, f_{1}, \ldots, f_{N-1}\right\}$, where $N$ is the length, can be presented in a vector matrix form as

$$
\alpha=\mathbf{W f}
$$

where $\boldsymbol{\alpha}$ contains $N$ wavelet coefficients, and $\mathbf{W}(N \times$ $N$ ) is an orthogonal matrix consisting of row basis vectors. The basis vector are specified by a set of numbers, called wavelet and scaling filter coefficients.

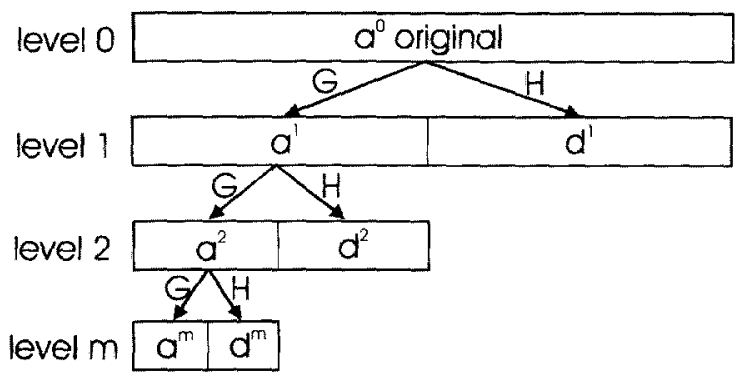

Fig. 1. Decomposition of $a^{0}$ up to level $m$ using DWT.

Once a specific wavelet has been chosen, we can use its coefficients to define two filters, the low-pass filter and the high-pass filter. Both types of filters use the same set of wavelet filter coefficients, but with alternating signs and in reversed order, meaning this pair of filters is the quadrature mirror filters (QMF). The lowpass and high-pass filters are also called the scaling and the wavelet filters, respectively. These filters are used to construct the filter matrices, denoted as $\mathbf{G}$ and $\mathbf{H}$.

To decompose (or analyze) the signal, Mallat (1989) introduced a recursive algorithm which is known as pyramid algorithm. This algorithm offers the hierarchical, multiresolution of the signal. In this algorithm the set of $N$ input data is passed through the low-pass and high-pass filters. Each output of the filter consists of $N / 2$ wavelet coefficients. The output from low-pass filter is the approximation coefficients $\left(a^{1}=\left\{a_{0}^{1}, a_{1}^{1}, \ldots, a_{N / 2-1}^{1}\right\}\right)$ at the first level of resolution. The output from high-pass filter is the detail coefficients $\left(\boldsymbol{d}^{1}=\left\{d_{0}^{1}, d_{1}^{1}, \ldots, d_{N / 2-1}^{1}\right\}\right)$ at the first level of resolution. The approximation coefficient $\boldsymbol{a}^{1}$, can now be used as the data input for another pair of wavelet filters (identical to the first pair), generating sets of length
$N / 4$ of approximation $\left(\boldsymbol{a}^{2}=\left\{a_{0}^{2}, a_{1}^{2}, \ldots, a_{N / 4-1}^{2}\right\}\right)$ and details coefficients $\left(\boldsymbol{d}^{2}=\left\{d_{0}^{2}, d_{1}^{2}, \ldots, d_{N / 4-1}^{2}\right\}\right)$ at the second level of resolution. The process is continued until a desired level of resolution. Since the original input data vector, $\boldsymbol{f}$, is the approximation at the lowest level of resolution (level 0), i.e.: $\boldsymbol{a}^{0}=\boldsymbol{f}=\left\{f_{0}, f_{1}, \ldots, f_{N-1}\right\}$, then the DWT algorithm can be presented by the following recursive formula

$$
\boldsymbol{a}^{m}=\mathbf{G} \boldsymbol{a}^{m-1} \quad \text { and } \quad \boldsymbol{d}^{m}=\mathbf{H} \boldsymbol{a}^{m-1}
$$

where $m$ denotes the resolution level and $m=1,2, \ldots$, $\log _{2} N$. Figure 1 shows this decomposition process.

The different resolution for each level is related to the sampling interval. For level $m$ the sampling interval equals $2^{m}$. As the sampling interval increases, resolution decreases and each approximation contains gradually less information. The difference in information between the approximations at level $m$ and level $m-1$ is contained in the detail at level $m$.

It is possible to use the approximation and detail coefficients to reconstruct (or synthesize) the original signal. The reconstruction process uses the recursion algorithm in reverse with conjugates of $\mathbf{G}$ and $\mathbf{H}$. For the orthonormal basis the conjugates of $\mathbf{G}$ and $\mathbf{H}$ equal to the transposed matrices $\mathbf{G}^{T}$ and $\mathbf{H}^{T}$, respectively. Thus, the reconstruction formula is as follows

$$
\boldsymbol{a}^{m-1}=\mathbf{G}^{T} \boldsymbol{a}^{m}+\mathbf{H}^{T} \boldsymbol{d}^{m} . \ldots \ldots \ldots \ldots \ldots \ldots
$$

\subsection{Wavelet Packet Transform}

2.2.1 Theory Wavelet packet transform is a direct expansion of the structure of the DWT tree algorithm to a full binary tree. In the pyramid algorithm the detail branches are not used for further calculations, only the approximations at each level of resolution are treated to yield approximation and detail obtained at higher level. For the wavelet packet, both the detail

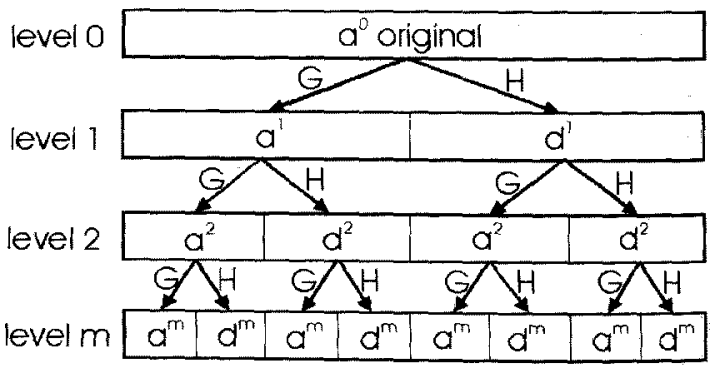

Fig. 2. Wavelet packet decomposition of $\boldsymbol{a}^{0}$ viewed as a binary tree.

and approximation coefficients at level $m$ are further decomposed into level $m+1$. The main advantage of the WPT is better signal representation. The search for the best representation of the signal by any subtree of the WPT is called the best-basis selection. Wavelet packet decomposition is shown in Fig. 2, in a tree structure to indicate the decomposition processes. The detail and approximation coefficients in each level for each tree (or subspace) are derived in similar manner to those of DWT using Eq.(2). 
2.2.2 Best-Basis Selection The complete signal representation by the WPT allows one to choose the appropriate representation of the signal. To find the best-basis or the wavelet coefficients of the besttree, one first computes its complete detail and approximation (wavelet) coefficients up to a desired level. Then, it is very natural to use the entropy as a measure of efficiency of the basis (Coifmann and Wickerhauser, 1992). Here the entropy of a signal $\boldsymbol{x}=\left\{x_{n}\right\}=$ $\left\{x_{0}, x_{1}, \ldots, x_{N-1}\right\}$ is defined as

$$
H(\boldsymbol{x})=-\sum_{n}^{N-1}\left|x_{n}\right|^{2} \log \left|x_{n}\right|^{2},
$$

which is known as the non-normalized Shannon entropy (Wickerhauser, 1994). The best-basis is the basis giving the minimum entropy or maximum information for its distribution of coefficients (Coifmann and Wickerhauser, 1992; Wickerhauser, 1994).

The wavelet packet may be efficiently searched for the best-basis. Each tree in the binary tree as shown in Fig. 2 represents a subspace, consisting of the detail or approximation coefficients, of the original signal. Each parent subspace is the orthogonal sum of its two children's subspaces. The search for the best-basis involves computing entropy using Eq.(4) for each subspace, then performing a comparison between the entropy of parent subspace and that of its two children's subspaces. If the parent has a smaller entropy, its two children are omitted from the tree. On the other hand, if the parent has a larger entropy, its two children are kept from the tree. This process is repeated until the original signal at the top level is reached (see also Fig. 4).

\section{Minimum Description Length Criterion}

The Minimum Description Length (MDL) criterion is an interesting approach to simultaneous noise suppression and signal compression. It is free from any parameter setting such as threshold selection, which can be particularly useful for real data where the noise level is difficult to estimate. The MDL criterion aims to gain the compromise between the number of retained wavelet coefficients and the error of signal reconstruction. This criterion selects the "best" wavelet filter and the "best" number of wavelet coefficients for the signal reconstruction (Saito, 1994).

The MDL criterion has the following algorithm. Let us consider a discrete model

$$
f=x+n
$$

where the vector $f$ represent the noisy observed data, vector $\boldsymbol{x}$ is the unknown true signal to be estimated, and vector $\boldsymbol{n}$ is noise. First, pick the index $(k, n)$ from the MDL function defined as

$M D L(k, n)=\min \left\{\frac{3}{2} k \log N+\frac{N}{2} \log \left\|\tilde{\boldsymbol{\alpha}}_{n}-\boldsymbol{\alpha}_{n}^{(k)}\right\|^{2}\right\}$

$0 \leq k<N ; 1 \leq n \leq M$

where $\tilde{\boldsymbol{\alpha}}_{n}=\mathbf{W}_{n} f$ denotes the vector of the sorted decomposition coefficients of $f$ via the wavelet filter $n$, and $\boldsymbol{\alpha}_{n}^{(k)}=\Theta^{(k)} \tilde{\boldsymbol{\alpha}}_{n}=\Theta^{(k)}\left(\mathbf{W}_{n} \boldsymbol{f}\right)$ denotes the vector that contains $k$ nonzero elements, and $\Theta^{(k)}$ is a hardthresholding operation which keeps the $k$ largest elements of $\tilde{\boldsymbol{\alpha}}_{n}$ in absolute value intact and set all other elements to zero. The $N$ and $M$ denote respectively the length of the signal and the total number of wavelet filters used. The $\tilde{\boldsymbol{\alpha}}_{n}$ and $\boldsymbol{\alpha}_{n}^{(k)}$ have to be normalized by $\left\|\tilde{\boldsymbol{\alpha}}_{n}\right\|$, so that the magnitude of each coefficient in $\tilde{\boldsymbol{\alpha}}_{n}$ and $\boldsymbol{\alpha}_{n}^{(k)}$ is strictly less than one. Note that $\|\boldsymbol{x}\|$ is defined as $\left(\sum_{0}^{N-1}\left|x_{n}\right|^{2}\right)^{1 / 2}$. The MDL function in Eq.(5) is expressed as the sum of two conflicting terms. The first term represents the penalty function, linearly increasing with the number of the retained wavelet coefficients $k$, whereas the second term describes the logarithmic of residual energy between $\tilde{\alpha}_{n}$ and $\boldsymbol{\alpha}_{n}^{(k)}$. We see that the $\log$ (residual energy) always decreases as $k$ increases (see also Fig. 5 later). Number of coefficients $k$, for which the MDL function reaches its minimum, is considered as the optimal one. With this criterion one can optimize the choice of wavelet filter as well. It
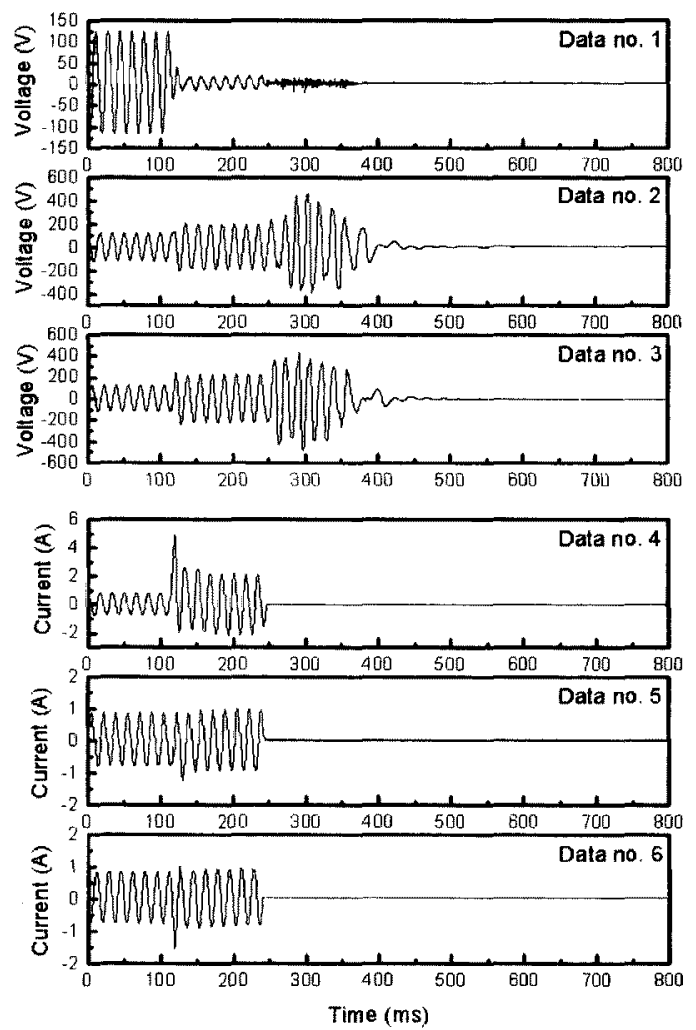

Fig. 3. Fault record from a single-phase to ground of three-phase power system. Data no. 1,2 and 3 are the voltage of phase $a$, phase $b$ and phase $c$, respectively, and the data 4,5 and 6 are for the current of phase $a$, phase $b$ and phase $c$, respectively. The fault occurred at $116 \mathrm{~ms}$ on phase a.

should be noted that each wavelet filter has different characteristics. A wavelet filter, which is optimal for a given signal, is not necessarily the best for another type 


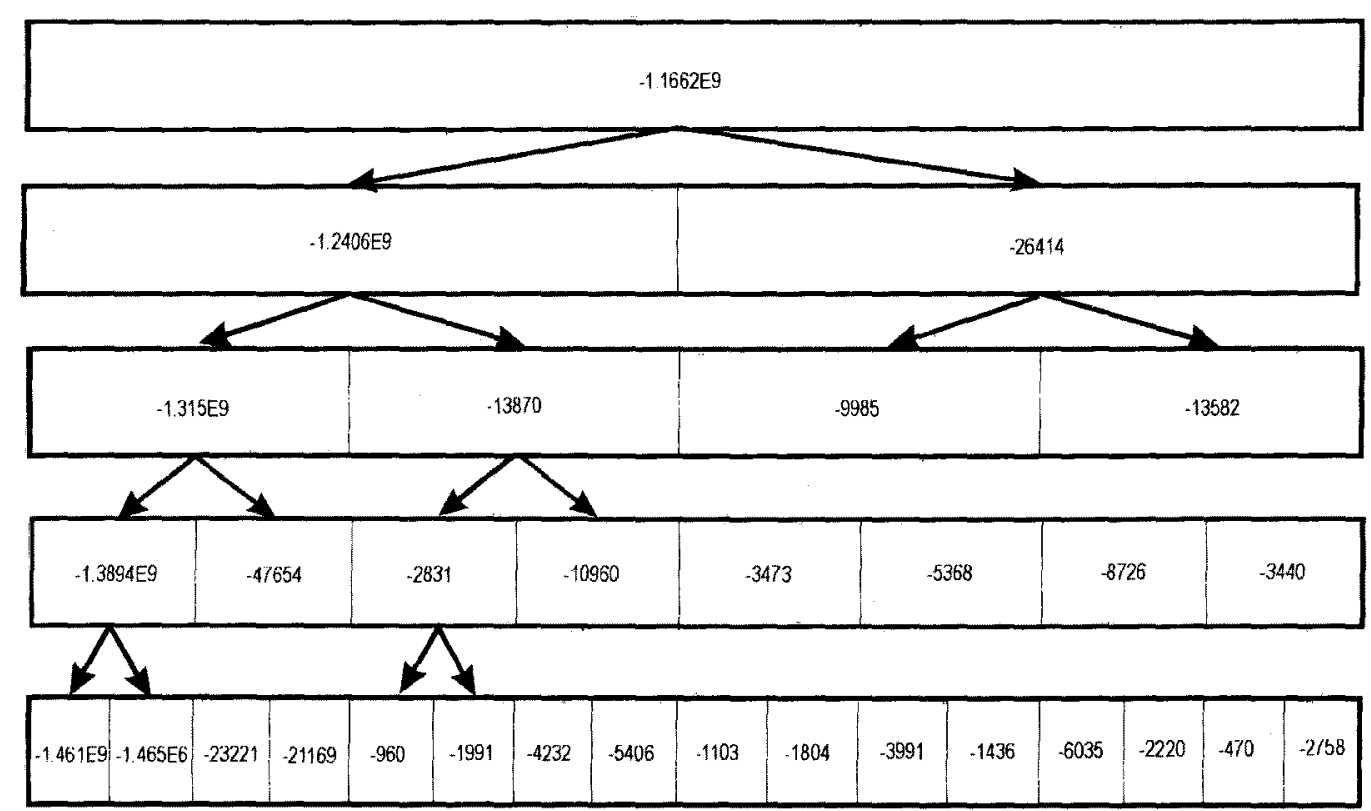

Fig. 4. The entropy value of each subspace, and the tree indicates the wavelet packet best-basis of data no. 2.

of signal.

Second, reconstruct the estimated true signal $x=$ $\left\{x_{n}\right\}=\left\{x_{0}, x_{1}, \ldots, x_{N-1}\right\}$ through the following equation

$$
\boldsymbol{x}=\mathbf{W}_{n}^{T} \boldsymbol{\alpha}_{n}^{(k)},
$$

which is exactly the same process as in Eq.(3).

\section{Experimental Study}

4.1 Power Disturbance Data The experimental study has been carried out for a single-phase to ground fault event, and six power disturbance data have been recorded. The data were obtained from a power system simulator (APSA: Advanced Power System Analyzer) owned by Kansai Electric Power Company (KEPCO), Japan. The performances of DWT and WPT compression are evaluated using these power disturbance data. Figure 3 shows these original signals. The length of each signal is $N=8000$ samples for 800 ms. Each sample requires 12 bytes ASCII and only the magnitudes are stored, so that each signal has a size of 96,000 bytes.

4.2 Library of Wavelet Filters Ten wavelets from the Daubechies family (with 2, 4, 6, 8, 10, 12, $14,16,18$, and 20 filter coefficients), five wavelets from Coiflets (with 2, 4, 6, 8, and 10 filter coefficients), and seven wavelets from Symlets (with $4,6,8,10,12,14$, and 16 filter coefficients) are used for the data compression. This corresponds to $M=22$. The coefficients of each wavelet filter can be found in Wickerhauser, (1994).

4.3 Performance Evaluation To evaluate the compression performance, two performance indexes are employed. The first one is the compression ratio (CR), i.e., the ratio of the size of the compressed file over the size of the original file, defined as

$$
C R(\%)=\frac{\text { bytes of the compressed signal }}{\text { bytes of the original signal }} \times 100 \text {. }
$$

The second one is the percentage of mean square error, defined as

$$
\operatorname{MSE}(\%)=\frac{\sqrt{\sum_{n=0}^{N-1}\left(f_{n}-x_{n}\right)^{2}}}{\sqrt{\sum_{n=0}^{N-1} f_{n}^{2}}} \times 100 \ldots \ldots
$$

where $f$ and $x$ are noisy observed (or original) signal and reconstructed signal, respectively.

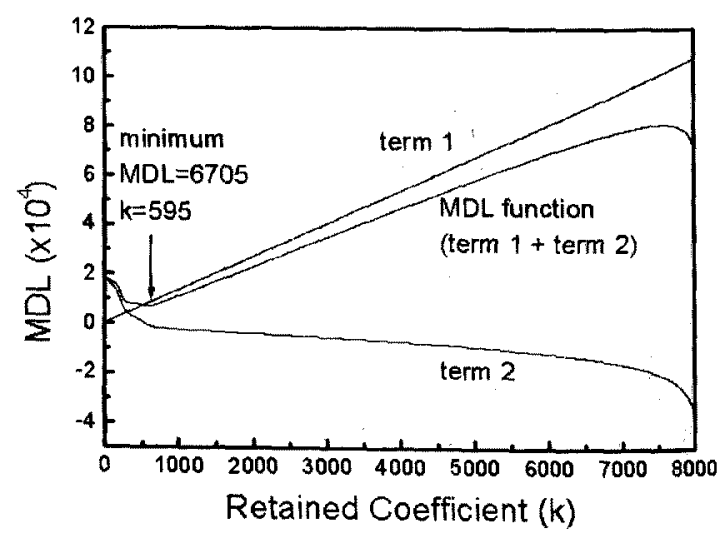

Fig. 5. The MDL function and its components for the WPT coefficients of data no. 2 with $\mathrm{Db} 5$ filter. 
Table 1. Number of retained coefficients, MSE, and MDL value for 22 wavelet filters using DWT

\begin{tabular}{|c|c|c|c|c|c|c|c|c|c|c|c|c|c|c|c|c|c|c|}
\hline $\begin{array}{c}\text { Filter } \\
n\end{array}$ & $\begin{array}{l}\mathrm{k} \\
\text { (1) }\end{array}$ & $\begin{array}{c}\text { MSE } \\
\text { (1) }\end{array}$ & $\begin{array}{c}\text { MDL } \\
\text { (1) }\end{array}$ & $\begin{array}{c}\mathrm{k} \\
(2)\end{array}$ & $\begin{array}{l}\text { MSE } \\
(2)\end{array}$ & $\begin{array}{c}\mathrm{MDL} \\
(2)\end{array}$ & $\begin{array}{c}\mathrm{k} \\
(3)\end{array}$ & $\begin{array}{l}\text { MSE } \\
(3)\end{array}$ & $\begin{array}{c}\text { MDL } \\
\text { (3) }\end{array}$ & $\begin{array}{l}k \\
(4)\end{array}$ & $\begin{array}{c}\text { MSE } \\
(4)\end{array}$ & $\begin{array}{c}\text { MDL } \\
\text { (4) }\end{array}$ & $\begin{array}{l}\mathrm{k} \\
(5)\end{array}$ & $\begin{array}{c}\text { MSE } \\
\text { (5) }\end{array}$ & $\begin{array}{l}\text { MDL } \\
\text { (5) }\end{array}$ & $\begin{array}{c}k \\
(6)\end{array}$ & $\begin{array}{c}\text { MSE } \\
(6)\end{array}$ & $\begin{array}{c}\text { MDL } \\
(6)\end{array}$ \\
\hline $\mathrm{Db1}$ & 160 & 14.68 & 12904 & 336 & 10.38 & 13891 & 327 & 10.31 & 13740 & 214 & 9.86 & 12041 & 247 & 11.00 & 12922 & 217 & 13.84 & 13435 \\
\hline $\mathrm{Db} 2$ & 133 & 10.95 & 11367 & 341 & 3.40 & 9491 & 388 & 2.76 & 9290 & 205 & 3.63 & 7916 & 168 & 6.56 & 9789 & 147 & 9.97 & 11182 \\
\hline Db3 & 135 & 10.13 & $11080^{*}$ & 598 & 0.89 & 7607 & 629 & 0.73 & 7246 & 163 & 3.26 & 6927 & 591 & 1.14 & 8478 & 556 & 1.72 & 9671 \\
\hline$\overline{\mathrm{Db} 4}$ & 138 & 10.11 & 11114 & 577 & 0.75 & 6644 & 600 & 0.62 & 6205 & 539 & 0.85 & 6638 & 547 & 1.15 & 7931 & 527 & 1.71 & 9246 \\
\hline Db5 & 138 & 10.17 & 11137 & 588 & 0.72 & 6611 & 609 & 0.59 & 6084 & 528 & 0.84 & $6434^{*}$ & 547 & 1.13 & 7845 & 529 & 1.64 & 9106 \\
\hline $\mathrm{Db} 6$ & 140 & 10.02 & 11105 & 577 & 0.73 & 6539 & 601 & 0.59 & 6016 & 537 & 0.83 & 6477 & 541 & 1.11 & 7693 & 533 & 1.61 & 9098 \\
\hline $\mathrm{Db} 7$ & 132 & 10.50 & 11184 & 593 & 0.72 & 6696 & 603 & 0.59 & 6041 & 537 & 0.82 & 6445 & 538 & 1.10 & $7629^{*}$ & 533 & 1.61 & 9091 \\
\hline $\mathrm{Db} 8$ & 144 & 10.12 & $\frac{1104}{11200}$ & 577 & 0.73 & 6537 & 604 & 0.60 & 6128 & 541 & $\begin{array}{l}0.02 \\
0.82\end{array}$ & 6490 & 547 & 1.08 & 7699 & 531 & 1.61 & $9065^{*}$ \\
\hline Db9 & 143 & 10.16 & 11200 & 583 & 0.74 & 6667 & 601 & 0.59 & $6004^{*}$ & 539 & 0.82 & 6482 & 548 & 1.08 & 7683 & 539 & 1.59 & 9118 \\
\hline $\mathrm{Db} 10$ & 144 & 10.20 & 11229 & 578 & 0.73 & 6550 & 608 & 0.59 & 6112 & 542 & 0.82 & 651 & 540 & 1.09 & $7631^{*}$ & 534 & 1.61 & 9115 \\
\hline Coif1 & 136 & 10.70 & 11313 & 331 & 3.49 & 9457 & 377 & 2.75 & 9125 & 201 & 3.67 & 7908 & 165 & 6.47 & 9695 & 633 & 1.92 & 11139 \\
\hline Coif2 & 147 & 9.92 & 11159 & 582 & 0.76 & 6758 & 597 & 0.63 & 6221 & 544 & 0.85 & 6704 & 551 & 1.13 & 7911 & 533 & 1.67 & 9232 \\
\hline Coif3 & 146 & 10.18 & 11248 & 577 & 0.75 & 6611 & 603 & $\begin{array}{l}0.60 \\
0.60\end{array}$ & 6068 & 541 & 0.83 & 6534 & 541 & 1.10 & 7683 & 535 & 1.63 & 9162 \\
\hline Coif4 & 151 & 10.11 & 11290 & 582 & 0.73 & 6589 & 610 & 0.59 & 61.15 & 545 & 0.82 & 6576 & 552 & 1.09 & 7782 & 540 & 1.60 & 9162 \\
\hline Coif5 & 161 & 9.92 & 11351 & 588 & 0.74 & 6713 & 602 & 0.61 & 611 & 556 & 0.82 & 6690 & 558 & 1.0 & 7780 & 544 & 1.60 & 9205 \\
\hline Sym2 & 133 & 10.95 & 11367 & 341 & 3.40 & 9491 & 388 & 2.76 & 9290 & 205 & 3.63 & 7916 & 168 & 6.5 & 9789 & 147 & 9.97 & 11182 \\
\hline Sym3 & 135 & 10.13 & 11080 & 598 & 0.89 & 7607 & 629 & 0.7 & 7246 & 163 & 3.26 & 6927 & 591 & 1.1 & 8478 & 556 & 1.72 & 9671 \\
\hline Sym4 & 138 & 9.99 & $11065^{*}$ & 578 & 0.77 & 6766 & 590 & 0.6 & 621 & 534 & 0.88 & 6692 & 545 & 1.1 & 7910 & 529 & 1.68 & 9217 \\
\hline Sym5 & 138 & 10.10 & 11111 & 584 & 0.72 & 6584 & 609 & 0.58 & 6054 & 537 & 0.84 & 6518 & 546 & 1.1 & 7828 & 532 & 1.63 & 9120 \\
\hline Sym6 & 141 & 10.02 & 11120 & 587 & 0.71 & 6561 & 601 & 0.59 & $\frac{6024}{604}$ & 536 & 0.83 & 6468 & 536 & 1.12 & 7664 & 528 & 1.62 & $9056^{*}$ \\
\hline Sym7 & 138 & 10.26 & 11175 & 569 & 0.75 & $6502^{*}$ & 603 & 0.59 & 6022 & 536 & 0.82 & $6443^{*}$ & 532 & 1.1 & 7637 & 537 & 1.60 & 9114 \\
\hline Sym8 & 142 & 10.20 & 11202 & 577 & 0.72 & $6486^{*}$ & 592 & 0.61 & $5971^{*}$ & 543 & 0.83 & 6554 & 543 & 1.09 & 7671 & 535 & 1.61 & 9114 \\
\hline
\end{tabular}

Table 2. Number of retained coefficients, MSE, and MDL value for 22 wavelet filters using WPT

\begin{tabular}{|c|c|c|c|c|c|c|c|c|c|c|c|c|c|c|c|c|c|c|}
\hline $\begin{array}{c}\text { Filter } \\
\text { n }\end{array}$ & $\begin{array}{c}k \\
(1)\end{array}$ & $\begin{array}{c}\text { MSE } \\
(1)\end{array}$ & $\begin{array}{l}\text { MDL } \\
\text { (1) }\end{array}$ & $\begin{array}{c}\mathbf{k} \\
(2)\end{array}$ & $\begin{array}{c}\text { MSE } \\
(2)\end{array}$ & $\begin{array}{c}\text { MDL } \\
\text { (2) }\end{array}$ & $\begin{array}{c}\mathrm{k} \\
(3)\end{array}$ & $\begin{array}{c}\text { MSE } \\
\text { (3) }\end{array}$ & $\begin{array}{c}\text { MDL } \\
\text { (3) }\end{array}$ & $\begin{array}{c}k \\
(4)\end{array}$ & $\begin{array}{c}\text { MSE } \\
(4)\end{array}$ & $\begin{array}{c}\mathrm{MDL} \\
(4)\end{array}$ & $\underset{(5)}{k}$ & $\begin{array}{c}\text { MSE } \\
(5)\end{array}$ & $\begin{array}{c}\text { MDL } \\
\text { (5) }\end{array}$ & $\begin{array}{c}\mathrm{k} \\
(6)\end{array}$ & $\begin{array}{c}\text { MSE } \\
(6)\end{array}$ & $\begin{array}{c}\text { MDL } \\
(6)\end{array}$ \\
\hline Db1 & 187 & 13.40 & 12890 & 341 & 9.98 & 13787 & 336 & 9.74 & 13622 & 352 & 5.46 & 11521 & 279 & 9.82 & 12886 & 217 & 13.88 & 13433 \\
\hline Db2 & 135 & 10.90 & 11362 & 354 & 3.26 & 9481 & 387 & 2.76 & 9261 & 206 & 3.63 & 7916 & 169 & 6.56 & 9789 & 148 & 9.97 & 11182 \\
\hline$\overline{\mathrm{Db} 3}$ & 139 & 10.05 & 11090 & 608 & 0.88 & 7670 & 628 & 0.75 & 7287 & 165 & 3.25 & 6926 & 592 & 1.14 & 8478 & 557 & 1.72 & 9671 \\
\hline $\mathrm{Db} 4$ & 142 & 10.06 & 11136 & 583 & 0.75 & 6705 & 603 & 0.65 & 6382 & 539 & 0.85 & 6621 & 550 & 1.15 & 7967 & 528 & 1.71 & 9239 \\
\hline Db5 & 135 & 10.44 & 11189 & 595 & 0.72 & 6705 & 620 & 0.58 & 6166 & 530 & 0.84 & $6453^{*}$ & 547 & 1.12 & 7816 & 529 & 1.64 & 9091 \\
\hline Db6 & 146 & 10.02 & 11173 & 593 & 0.71 & 6626 & 593 & 0.62 & 6060 & 538 & 0.83 & 6480 & 540 & 1.12 & 7704 & 532 & 1.62 & 9082 \\
\hline Db7 & 138 & 10.50 & 11251 & 589 & 0.75 & 6766 & 594 & 0.63 & 6125 & 538 & 0.83 & 6480 & 537 & 1.10 & $7625^{*}$ & 534 & 1.61 & 9079 \\
\hline Db8 & 150 & 10.18 & 11291 & 595 & 0.71 & 6631 & 614 & 0.59 & 6149 & 541 & 0.82 & 6487 & 549 & 1.08 & 7707 & 531 & 1.61 & $9055^{*}$ \\
\hline Db9 & 129 & 10.27 & $11044^{*}$ & 587 & 0.74 & 6712 & 596 & 0.60 & 6007 & 540 & 0.82 & 6482 & 549 & 1.08 & 7683 & 540 & 1.59 & 9117 \\
\hline Db10 & 137 & 10.29 & 11158 & 587 & 0.73 & 6664 & 603 & 0.60 & 6072 & 543 & 0.82 & 6516 & 541 & 1.09 & 7631 & 535 & 1.61 & 9115 \\
\hline Coif1 & 136 & 10.73 & 11313 & 331 & 3.50 & 9463 & 381 & 2.73 & 9137 & 202 & 3.67 & 7908 & 166 & 6.47 & 9695 & 634 & t.92 & $1 \overline{1147}$ \\
\hline Coif2 & 148 & 9.92 & 11159 & 586 & $0 . \overline{74}$ & 6708 & 597 & 0.63 & 6193 & 541 & 0.86 & 6658 & 549 & 1.14 & 7910 & 534 & 1.66 & 9209 \\
\hline Coif3 & 150 & 10.01 & 11221 & 585 & 0.71 & 6492 & 600 & 0.59 & 5964 & 540 & 0.83 & 6510 & 539 & 1.10 & 7652 & 534 & 1.63 & 9138 \\
\hline Coif4 & 154 & 10.03 & 11286 & 586 & 0.70 & 6487 & 611 & 0.58 & 6028 & 546 & 0.82 & 6576 & 553 & 1.09 & 7782 & 541 & 1.60 & 9162 \\
\hline Coif5 & 162 & 9.94 & 11357 & 597 & 0.71 & 6657 & 601 & 0.59 & 5974 & 557 & 0.82 & 6690 & 559 & 1.07 & 7780 & 545 & 1.60 & 9205 \\
\hline Sym2 & 135 & 10.90 & 11362 & 354 & 3.26 & 9481 & 387 & 2.76 & 9261 & 206 & 3.63 & 7916 & 169 & 6.56 & 9789 & 148 & 9.97 & 11182 \\
\hline Sym3 & 139 & 10.05 & 11090 & 608 & 0.88 & 7670 & 628 & 0.75 & 7287 & 165 & 3.25 & 6926 & 592 & 1.14 & 8478 & 557 & 1.72 & 9671 \\
\hline Sym4 & 140 & 9.93 & $11058^{*}$ & 589 & 0.75 & 6752 & 591 & 0.65 & 6220 & 532 & 0.89 & 6681 & 544 & 1.15 & 7887 & 531 & 1.67 & 9199 \\
\hline Sym5 & 140 & 10.05 & 11103 & 586 & 0.72 & 6590 & 593 & 0.61 & 6033 & 539 & 0.8 .3 & 6524 & 545 & 1.13 & 7807 & 530 & 1.64 & 9101 \\
\hline Sym6 & 144 & 9.96 & 11121 & 585 & 0.70 & 6459 & 599 & 0.59 & 5946 & 536 & 0.83 & 6474 & 537 & 1.12 & 7664 & 529 & 1.62 & $9056^{*}$ \\
\hline Sym7 & 146 & 10.01 & 11167 & 569 & 0.73 & $6420^{*}$ & 598 & 0.58 & $5885^{*}$ & 538 & 0.82 & $6464^{*}$ & 532 & 1.12 & $7610^{*}$ & 536 & 1.60 & 9093 \\
\hline Sym8 & 143 & 10.20 & 11203 & 580 & 0.71 & $6435^{*}$ & 583 & 0.60 & $5818^{*}$ & 543 & 0.83 & 6539 & 540 & 1.10 & 7647 & 535 & 1.60 & 9088 \\
\hline
\end{tabular}

Note: The number inside the parenthesis is the data number, and the asterisk $\left(^{*}\right)$ indicates the first two minimum MDL.

\section{Results}

We compare the performance of 22 wavelet filters for the compression. All signals are decomposed via the DWT and WPT with those filters up to fourth level of resolution $(m=4)$. For the case of the WPT, the decomposition is performed following the best-basis selection with minimum entropy criterion. The wavelet coefficients from the decomposition is sorted according to their absolute amplitude. The optimal number of retained coefficients $k$ can be calculated based on the MDL criterion.

To simplify the explanation we will give attention on the signal of data no. 2, and we apply the WPT with the Daubechies 5 (Db5) filter. First the data is decomposed up to a predefined level using Eq.(2). The entropy of each subspace is then calculated using Eq. (4) to find the best-basis. Figure 4 shows the result of the best-basis
Table 3. The appropriate wavelet filters based on MDL criterion

\begin{tabular}{|c|c|c|}
\hline Data & DWT & WPT \\
\hline 1 & Sym4 - Db3 & Db9 - Sym4 \\
\hline 2 & Sym8 - Sym7 & Sym7 - Sym8 \\
\hline 3 & Sym8- Db9 & Sym8- Sym7 \\
\hline 4 & Db5 - Sym7 & Db5 - Sym7 \\
\hline 5 & Db7 - Db10 & Sym7 - Db7 \\
\hline 6 & Sym6-Db8 & Db8 - Sym6 \\
\hline
\end{tabular}

with minimum entropy criterion. Once the best-basis is found the MDL function is applied to compute the number of wavelet retained coefficients $k$. The result of the MDL function and its components is shown in Fig. 5. The function reaches the minimum at $k=595$, meaning the number of coefficients required for the signal reconstruction using Db5 filter is 595 . The process 
above is repeated until the last wavelet filter in the library ( $n=M=22$ ), and then by selecting the lowest MDL value, the appropriate filter can be chosen.

Table 4. CR and MSE using DWT with Symlets 7 filter and lossless codings

\begin{tabular}{|c|c|c|c|c|c|}
\hline Data & $\begin{array}{c}\text { DWT } \\
(\%)\end{array}$ & $\begin{array}{c}\text { DWT+Huff. } \\
(\%)\end{array}$ & $\begin{array}{c}\text { DWT+LZW } \\
(\%)\end{array}$ & $\begin{array}{c}\text { DWT+LZH } \\
(\%)\end{array}$ & $\begin{array}{c}\text { MSE } \\
(\%)\end{array}$ \\
\hline 1 & 2.49 & 1.10 & 1.20 & 1.09 & 10.26 \\
\hline 2 & 10.10 & 4.38 & 4.45 & 4.19 & 0.75 \\
\hline 3 & 10.72 & 4.65 & 4.67 & 4.40 & 0.59 \\
\hline 4 & 9.54 & 3.74 & 3.20 & 2.80 & 0.82 \\
\hline 5 & 9.46 & 3.73 & 3.05 & 2.68 & 1.12 \\
\hline 6 & 9.55 & 3.76 & 3.10 & 2.74 & 1.60 \\
\hline
\end{tabular}

Table 5. CR and MSE using WPT with Symlets 7

filter and lossless codings

\begin{tabular}{|c|c|c|c|c|c|}
\hline Data & $\begin{array}{c}\text { WPT } \\
(\%)\end{array}$ & $\begin{array}{c}\text { WPT+Huff. } \\
(\%)\end{array}$ & $\begin{array}{c}\text { WPT+LZW } \\
(\%)\end{array}$ & $\begin{array}{c}\text { WPT+LZH } \\
(\%)\end{array}$ & $\begin{array}{c}\text { MSE } \\
(\%)\end{array}$ \\
\hline 1 & 2.75 & 1.21 & 1.32 & 1.19 & 10.01 \\
\hline 2 & 10.23 & 4.43 & 4.50 & 4.22 & 0.73 \\
\hline 3 & 10.75 & 4.66 & 4.67 & 4.39 & 0.58 \\
\hline 4 & 9.72 & 3.80 & 3.25 & 2.85 & 0.82 \\
\hline 5 & 9.55 & 3.76 & 3.07 & 2.70 & 1.12 \\
\hline 6 & 9.64 & 3.80 & 3.13 & 2.78 & 1.60 \\
\hline
\end{tabular}

We have applied the MDL criterion to all data to select the suitable filter, and the results are tabulated in Table 1 and Table 2 for the DWT and WPT, respectively. Both tables show the number of retained coefficients $k$, the MSE and the minimum value of the MDL function for all wavelet filters. From this point, we can chose the appropriate filter for each corresponding data based on the minimum MDL value, and the results for the first two filters having smallest MDL are tabulated in Table 3 . We can see that the appropriate filter for a given signal may different for another type of signal. However, in practice it is preferable to use only one "best" filter for all signals. From the table the Symlets 7 and Symlets 8 filters seem to be the candidates for the best filter. We simply select the Symlets 7 filter for the compression of all power disturbance data analyzed here.

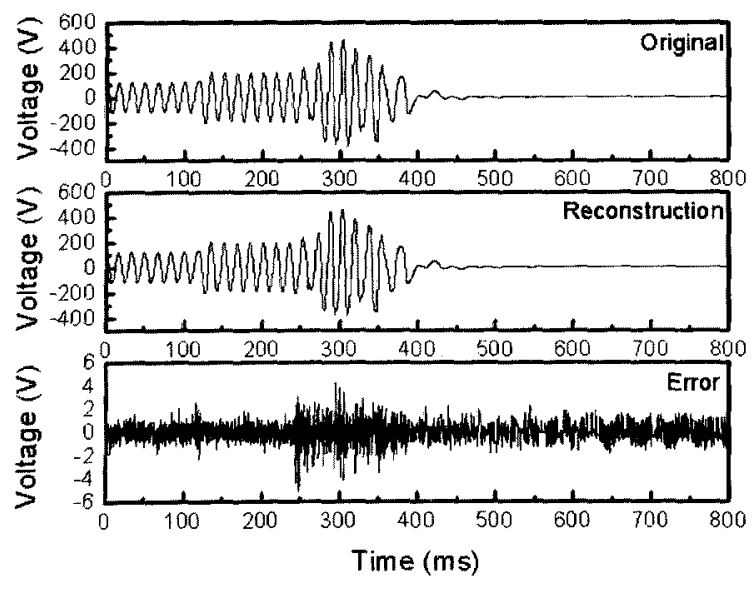

Fig. 6. The original, reconstructed, and residual error signals of data no. 2 using WPT with Sym7 filter.
Using the MDL we can compute the number of nonzero ratained coefficients to be stored as the compressed data. Here the compressed data contains both magnitude and position of those retained coefficients. We allocate 12 bytes ASCII for the magnitude and 5 bytes ASCII for its position.

In addition, more effective compression can be performed by implementing an additional lossless coding (e.g. Huffman, LZW, or LZH) to the results of the DWT and WPT compression. Here, we used the term "coded data" to the result of coding process. Since the coding has lossless properties; the coding result always reproduce the same data when a file is decoded. Table 4 and Table 5 show the comparison of CR and MSE of the analyzed signals using the Symlets 7 filter. The compressed file size (in percentage of original file size) is calculated for the DWT, WPT, and DWT+lossless coding as well as WPT+lossless coding. Both the DWT and WPT compression significantly reduce the original file size of each signal to less than $11 \%$. Further, the tables show that by implementing the lossless coding the CR's are reduced to more than a half of those CR's without the lossless coding.

For the signal reconstruction, first the coded data (the original data which is compressed via wavelet and lossless coding) is decoded. This decoded data contains non-zero wavelet retained coefficients and their location. Second, these wavelet retained coefficients are rearranged according to their locations, and then zero magnitudes are inserted to the rest of locations. Last, the signal reconstruction from these coefficients is done using Eq.(3). Figure 6 shows an example of the original signal, reconstructed signal and its residual error for data no. 2 using the Symlets 7 filter.

\section{Conclusions}

The application of the DWT and WPT to compress the data of power system disturbances has been demonstrated and evaluated. Both transforms offer attractive properties for the compression. The experimental results show that better quality reconstruction can be achieved by employing an appropriate wavelet filter to each signal. In practice, it is preferable to use one suitable filter for compressing all signals. Using the MDL criterion, the Symlets 7 filter generally appears superior than other wavelet filters for most power disturbance signals analyzed here. The compression ratios that can be obtained using this filter are varied but less than $11 \%$. Combining wavelet-based compression with a lossless coding could results in better compression ratios. Our results show that the compression ratios are reduced to more than a half by implementing an additional lossless coding. Finally, the compression algorithm presented here can be used to compress not only ground fault signals but also wide variety of onedimensional power system disturbance signals.

(Manuscript received January 22, 2001) 


\section{References}

(1) Coifmann, R. R., and M. V. Wickerhauser, Entropy-based algorithms for best basis selection, IEEE Trans. Inform. Theory, 38, 713-719, 1992

(2) Hilton, M. L., Wavelet and wavelet packet compression of electrocardiograms, IEEE Trans. Biomed. Eng., 44, 394-402, 1997.

(3) Littler, T. B., and D. J. Morrow, Wavelet for the analysis and compression of power system disturbances, IEEE Trans. Power Delivery, 14, 358-362, 1999.

(4) Mallat, S. A., A theory for multiresolution signal decomposition: The wavelet representation, IEEE Trans. Pattern Anal Machine Intell., 11, 674-693, 1989.

(5) Mehta, K. B., and B. D. Russel, Data Compression for digital data from power system disturbance: requirements and technique evaluation, IEEE Trans. Power Delivery, 4, 1683-1689, 1989.

(6) Saito, N., Simultaneous noise suppression and signal compression using a library of orthonormal bases and the minimum description length criterion, in Wavelets in Geophysic, E. Foufoula-Georgiou and P. Kumar (Eds), Academic Press, San Diego, CA, 299-324, 1994

(7) Santoso, S., E. J. Power, and W. M. Grady, Power quality data compression using wavelet transform methods, IEEE Trans. Power Delivery, 12, 1250-1257, 1997.

(8) Walczak, B., and D. L. Massart, Noise suppresion and signal compression using the wavelet packet transform, Chemom. Intel. Lab. Sys., 36, 81-94, 1997.

(9) Wickerhauser, M. V., Adapted wavelet analysis from theory to software, IEEE Press, New York, USA, 273-298 and 443$462,1994$.

Effrina Yanti Hamid (Non-member) She was born in In-

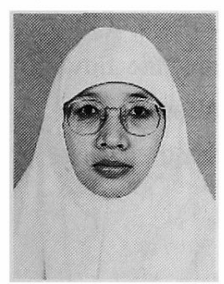
donesia in 1972. She received the bachelor and master degrees in electrical engineering from Bandung Institute of Technology (ITB), Indonesia in 1995 and 1998, respectively. Now, she is a Ph.D student in the Department of Electrical Engineering, Osaka University. Her main interest is in signal processing and its application to power system.

Zen-Ichiro Kawasaki (Member) He was born in Japan in

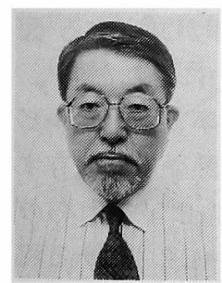
1949. He received the B.S., M.S. and Dr. Eng. degrees in communication engineering from Osaka University, Japan in 1973, 1975 and 1978 , respectively. In 1989 , he joined the Department of Electrical Engineering, Osaka University. Currently, he is a Professor at the Department of Communication Engineering in the same university. His current research interests are in signal processing, diagnosis techniques of power apparatus, and the electromagnetic of lightning discharges. Dr. Zen-Ichiro Kawasaki is a member of IEEE, IEE of Japan, American Geophysical Union (AGU), and The Society of Atmospheric Electricity of Japan (SAEJ).

Hirotaka Yoshida (Member) He received M.S. degree in

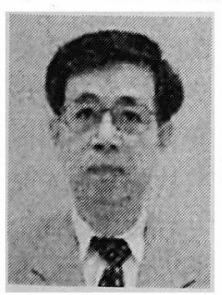
electrical engineering in 1976 from Doshish University, Kyoto, Japan. He has been working at The Kansai Electric Power Company from 1976. His research interests include analysis, planning, and operation of power systems. He is a member of IEE of Japan.
Hirosuke Doi (Member) He graduated an electric course of Technical High School in Japan, in 1965. He had joined The Kansai Electric Power Company, Japan. He has been working at Kansai Electric Instrument Company. He was commended to the persons of scientific and technological research merits by the Minister of State for Science anf Technology in 1991. He was awarded for the Technical Advancement from IEE in 1991 . He is a member of IEE of Japan. 